\title{
Condiciones laborales y de salud en trabajadores inmigrantes internacionales
}

\section{Working and health conditions in international immigrant workers}

\author{
Ikram Benazizi ${ }^{1,2}$ \\ 1Departamento de Salud Pública, Historia de la Ciencia y Ginecología de la Universidad Miguel Her- \\ nández de Elche. \\ ${ }^{2}$ Grupo de Investigación en Salud Pública de la Universidad de Alicante.
}

\section{Resumen}

Este trabajo es un comentario del artículo: Hargreaves S, Rustage K, Nellums LB, McAlpine A, Pocock N, Devakumar D, Aldridge RW, Abubakar I, Kristensen KL, Himmels JW, Friedland JS, Zimmerman C. Occupational health outcomes among international migrant workers: a systematic review and meta-analysis. The Lancet Global Health. 2019; 7(7): E872-82. doi: https://doi.org/10.1016/S2214-109X(19)30204-9

\section{Abstract}

This text is a commentary on the article: Hargreaves S, Rustage K, Nellums LB, McAlpine A, Pocock N, Devakumar D, Aldridge RW, Abubakar I, Kristensen KL, Himmels JW, Friedland JS, Zimmerman C. Occupational health outcomes among international migrant workers: a systematic review and meta-analysis. The Lancet Global Health. 2019; 7(7): E872-82. doi: https://doi.org/10.1016/S2214-09X(19)30204-9

Sección coordinada por

Consol Serra (consol.serra@upf.edu) | Mª del Mar Seguí (mm.segui@ua.es)

Fechas · Dates

Recibido: 2020.12.18

Aceptado: 2020.12 .18

Publicado: 2021.01.21
Correspondencia · Corresponding Author

Ikram Benazizi

Departamento de Salud Pública, Historia de la Ciencia y Ginecología de la Universidad Miguel Hernández de Elche. Grupo de Investigación en Salud Pública de la Universidad de Alicante. ibenazizi@umh.es 


\section{Resumen del artículo comentado}

En el mundo hay más de 150 millones de trabajadores migrantes internacionales, personas que están empleadas fuera de su país de origen, que componen el grupo de migrantes internacionales más grande. Un número considerable de migrantes trabaja en entornos peligrosos y de explotación, donde podrían correr un riesgo considerable de sufrir lesiones y problemas de salud. Sin embargo, existen pocos datos sobre los resultados de la salud ocupacional de los trabajadores migrantes que sirvan de base para la formulación de políticas mundiales y la prestación de servicios de salud.

En esta revisión sistemática y metaanálisis, se realizaron búsquedas en las bases de datos Embase, MEDLINE, Ovid Global Health y PsychINFO para identificar estudios originales publicados entre el 1 de enero de 2008 y el 24 de enero de 2018, que informaran de resultados de salud ocupacional en trabajadores migrantes internacionales (definidos como individuos que están o han sido empleados fuera de su país de origen), sin restricciones de idioma o geográficas. Se excluyeron los estudios que contenían cohortes mixtas de migrantes y trabajadores nativos en los que los datos sobre migrantes no podían desglosarse y los que no informaban explícitamente sobre la condición de migrante. El resultado principal fue la prevalencia de los resultados de salud ocupacional (definidos como cualquier lesión, mortalidad o morbilidad física o psiquiátrica debido al trabajo o al entorno laboral de una persona) entre los trabajadores migrantes internacionales. Las estimaciones resumidas se calcularon utilizando modelos de efectos aleatorios. El protocolo del estudio ha sido registrado en PROSPERO, con número CRD42018099465.

De los 1218 estudios identificados en nuestra búsqueda, 36 estudios se incluyeron en la revisión sistemática y 18 en el metaanálisis. La revisión sistemática incluyó resultados de salud ocupacional para 12168 trabajadores migrantes internacionales empleados en 13 países y territorios, en su mayoría empleados en trabajo manual no cualificado. Los trabajadores migrantes procedían de 25 países de ingresos bajos y medios y trabajaban en los siguientes sectores: agricultura; sector doméstico, minorista y de servicios; construcción y comercio; y fabricación y procesamiento. Los trabajadores migrantes padecían diversas enfermedades psiquiátricas y físicas, y los accidentes y lesiones en el lugar de trabajo eran relativamente comunes. En el metaanálisis, entre 7260 trabajadores migrantes internacionales, la prevalencia combinada de tener al menos una morbilidad ocupacional fue del $47 \%\left(95 \% \mathrm{Cl} 29-64 ;\left.\right|^{2}=99 \cdot 70 \%\right)$. Entre 3890 trabajadores migrantes, la prevalencia de sufrir al menos una lesión o accidente, incluidas caídas de altura, fracturas y dislocaciones, lesiones oculares y cortes, fue del $22 \%\left(7-37 ;\left.\right|^{2}=99 \cdot 35 \%\right)$.

Los trabajadores migrantes internacionales presentan un riesgo considerable de sufrir lesiones y enfermedades relacionadas con el trabajo, y sus necesidades de salud se pasan por alto en la investigación y en política. Los gobiernos, los formuladores de políticas y las empresas deben hacer cumplir y mejorar las medidas de seguridad y salud ocupacional, que deben ir acompañadas de una cobertura de seguro y atención médica accesible, asequible y adecuada para satisfacer las necesidades de atención de esta importante población activa. 


\section{Comentario}

Los cambios en las condiciones de empleo en la economía mundial durante las últimas décadas han provocado una mayor inseguridad laboral y otros riesgos para la organización del trabajo y además se han visto agravados de manera considerable por la pandemia actual de COVID-19(1). Estos riesgos pueden influir en la aparición y el mantenimiento de disparidades de salud laboral por estatus migratorio ${ }^{(2)}$. Los trabajadores inmigrantes constituyen un sector en crecimiento de la fuerza laboral sobre todo en los países de ingresos medio-altos, y a nivel mundial representan una proporción importante de trabjadores ${ }^{(3)}$.

En comparación con los trabajadores autóctonos, se ha descrito que los inmigrantes muestran mayor prevalencia de trabajos manuales o poco cualificados, empleos temporales o informales, bajos salarios con largas horas de trabajo y discriminación percibida en el trabajo. Asimismo, experimentan mayores exigencias físicas, peores condiciones ambientales de trabajo y mayor exposición a los riesgos laborales, probablemente porque estén empleados en trabajos e industrias más peligrosos, por lo que presentan tasas más altas de lesiones ocupacionales y muerte ${ }^{(4)}$. La naturaleza precaria de sus empleos a menudo conlleva resultados de salud negativos y una peor salud general y mental, con alta carga de trastornos mentales comunes como la depresión; así como un posible empeoramiento de su estado de salud y una calidad de vida más baja que los autóctonos ${ }^{(5-6)}$. El estatus legal, las dificultades idiomáticas y el nivel de alfabetización juegan un papel determinante y pueden ser barreras que dificultan el control de los trabajadores inmigrantes sobre su entorno y condiciones de empleo limitando el acceso a una formación y capacitación adecuada y adaptada en materia de salud y seguridad ocupacional, así como al acceso a la atención médica ${ }^{(7-9)}$.

La Convención internacional sobre la protección de los derechos de todos los trabajadores migratorios y de sus familiares de la Organización de las Naciones Unidas no se encuentra ratificada en ningún país de los principales beneficiarios de la mano de obra de trabajadores inmigrantes provenientes de países de rentas medio-bajas. Por ello, más países deberían ratificarla para brindar equidad en la salud mundial y garantizar que la salud de los trabajadores migrantes no se descuide(6).

Esta revisión sistemática publicada en "The Lancet Global Health" en mayo de 2019 proporciona un resumen completo de la carga de morbilidad y lesiones ocupacionales entre los trabajadores migrantes internacionales y demuestra los factores de riesgo ocupacional persistentes y los resultados de salud deficientes en este importante grupo de población trabajadora. Este trabajo pone de manifiesto altas tasas de morbilidad física y psiquiátrica, lesiones y accidentes entre los trabajadores migrantes internacionales, provenientes mayoritariamente de países de bajos y medios ingresos y empleados en trabajos manuales no cualificados, con salarios bajos y largas horas de trabajo. También destaca los derechos laborales limitados, el acceso restringido a la atención médica y el bienestar social y la dificultad de aculturación, así como los determinantes sociales relacionados con las condiciones de vida. Por ello, los trabajadores migrantes internacionales continúan teniendo un riesgo considerable de sufrir daños, problemas de salud 
relacionados con su entorno laboral, una salud autopercibida deficiente y otros problemas de salud.

Asimismo, en esta revisión se observa que los resultados en la salud de las personas trabajadoras varían en función del sector ocupacional. Las personas trabajadoras inmigrantes del sector de la agricultura o la construcción presentaron más probabilidades de sufrir un accidente o lesión en el trabajo que las empleadas en otras profesiones y, entre ellas, se dio con mayor frecuencia dolor musculoesquelético asociado a edad avanzada, largas horas de trabajo, postura de trabajo, y un clima de seguridad laboral deficiente. También eran frecuentes las infecciones dermatológicas atribuibles al calzado oclusivo usado por los trabajadores agrícolas y asociado con una edad más joven y sexo masculino, y la depresión fue otro resultado en la salud de las personas trabajadores en este sector, asociada con un aumento de la somnolencia diurna y el número de años empleados.

Entre los trabajadores domésticos, minoristas y empleados del sector de servicios informaron resultados asociados con la depresión relacionada con el efecto perjudicial del trabajo doméstico de cuidados internos sobre la calidad y el tiempo del sueño, estrés, abuso y aislamiento social asociados con un empeoramiento de la calidad de vida, y otros problemas psiquiátricos, además de los resultados físicos debido a la fatiga y las malas condiciones de trabajo.

Los resultados de salud entre los trabajadores de la construcción y el comercio incluyeron morbilidad psiquiátrica. Los dolores corporales y articulares y las lesiones eran comunes. En los trabajadores en industrias de fabricación y procesamiento los resultados en salud reflejaban dolor musculoesquelético, estrés relacionado con el trabajo y salud y bienestar autoinformados deficientes, relacionados con factores como la mayor edad, las largas horas y la gran carga de trabajo, la postura incómoda, el historial de lesiones, y la maquinaria problemática y poco fiable, así como con el estado civil y el número de personas dependientes de la persona trabajadora.

La revisión y el metaanálisis realizados destacaron que los trabajadores migrantes internacionales continúan teniendo un riesgo considerable de sufrir daños y problemas de salud como consecuencia de su entorno laboral. Otros factores también pueden estar asociados a los malos resultados de salud autoinformados entre los migrantes como derechos laborales limitados, acceso restringido a la atención médica y el bienestar social en los países de destino, y dificultades de aculturación y adaptación a los países de acogida. A su vez, los determinantes sociales como la vivienda y las condiciones de vida deficientes podrían contribuir al riesgo de sufrir otros problemas de salud.

Es destacable la influencia del acoso, la explotación, la violencia o la discriminación en los resultados de salud deficientes, en particular en la salud mental. La importancia de la integración a distintos niveles ya sea en los servicios de salud, en el mercado laboral, así como la integración social, también es un predictor de la salud de la población inmigrante.

Cabe discutir y reflejar la dificultad de registrar datos precisos al disponer de escasa información sobre la frecuencia con la que los inmigrantes informan sobre sus 
necesidades de salud ocupacional, por lo que los datos podrían estar infravalorados y subnotificados en morbilidad y mortalidad.

A pesar de ello, la alta prevalencia de morbilidad, lesiones y accidentes refuerza el hecho de tener un mayor avance hacia la cobertura universal de salud y la garantía del cumplimiento de los derechos de todos los trabajadores, especialmente los inmigrantes y teniendo en cuenta factores como la situación legal, el género, el tiempo de residencia y la ocupación, entre otros. La revisión refleja la necesidad de cumplimiento, mejora y adaptación de las medidas de seguridad y salud ocupacional para proteger los derechos y satisfacer las necesidades de atención de la población de trabajadores inmigrantes internacionales.

\section{Bibliografía}

1. Reid A, Ronda-Perez E, Schenker MB. Migrant workers, essential work, and COVID-19. Am J Ind Med. 2021;64(2):73-77.

2. Landsbergis PA, Grzywacz JG, LaMontagne AD. Work organization, job insecurity, and occupational health disparities. Am J Ind Med. 2014;57(5):495-515.

3. International Labour Organization. Report: Global Estimates on International Migrant Workers - Results and Methodology. [Citado 16 Dic 2020]. Disponible en: https://www.ilo.org/global/publications/books/WCMS_652001/lang--en/index.htm

4. Negi NJ, Swanberg JE, Clouser JM, Harmon-Darrow C. Working under conditions of social vulnerability: Depression among Latina/o immigrant horse workers. Cultur Divers Ethnic Minor Psychol. 2020;26(1):54-60.

5. Moyce SC, Schenker M. Occupational Exposures and Health Outcomes Among Immigrants in the USA. Curr Environ Health Rep. 2017; 4(3):349-54.

6. Arici C, Ronda-Pérez E, Tamhid T, Absekava K, Porru S. Occupational Health and Safety of Immigrant Workers in Italy and Spain: A Scoping Review. Int J Environ Res Public Health. 2019;16 (22): 4416.

7. Liem A, Wang C, Wariyanti Y, Latkin CA, Hall BJ. The neglected health of international migrant workers in the COVID-19 epidemic. Lancet Psychiatry. 2020;7 (4):e20.

8. Ramos AK, Carlo G, Grant K, Trinidad N, Correa A. Stress, Depression, and Occupational Injury among Migrant Farmworkers in Nebraska. Saf Basel Switz. 2016;2(4):23.

9. Reid A, Schenker MB. Hired farmworkers in the US: Demographics, work organisation, and services. Am J Ind Med. 2016;59(8):644-55.

10. Panikkar B, Woodin MA, Brugge D, Desmarais AM, Hyatt R, Community Partners of the Somerville Community Immigrant Worker Project, et al. Occupational health outcomes among self-identified immigrant workers living and working in Somerville, Massachusetts 2006-2009. J Immigr Minor Health. 2013;15(5):882-9. 